\title{
Least Squares Support Vector Machine Classifiers
}

\author{
J.A.K. SUYKENS and J. VANDEWALLE \\ Katholieke Universiteit Leuven, Department of Electrical Engineering, ESAT-SISTA Kardinaal \\ Mercierlaan 94, B-3001 Leuven (Heverlee),Belgium, e-mail: johan.suykens@esat.kuleuven.ac.be
}

\begin{abstract}
In this letter we discuss a least squares version for support vector machine (SVM) classifiers. Due to equality type constraints in the formulation, the solution follows from solving a set of linear equations, instead of quadratic programming for classical SVM's. The approach is illustrated on a two-spiral benchmark classification problem.
\end{abstract}

Key words: classification, support vector machines, linear least squares, radial basis function kernel

Abbreviations: SVM - Support Vector Machines; VC - Vapnik-Chervonenkis; RBF - Radial Basis Function

\section{Introduction}

Recently, support vector machines (Vapnik, 1995; Vapknik, 1998a; Vapnik, 1998b) have been introduced for solving pattern recognition problems. In this method one maps the data into a higher dimensional input space and one constructs an optimal separating hyperplane in this space. This basically involves solving a quadratic programming problem, while gradient based training methods for neural network architectures on the other hand suffer from the existence of many local minima (Bishop, 1995; Cherkassky \& Mulier, 1998; Haykin, 1994; Zurada, 1992). Kernel functions and parameters are chosen such that a bound on the $\mathrm{VC}$ dimension is minimized. Later, the support vector method was extended for solving function estimation problems. For this purpose Vapnik's epsilon insensitive loss function and Huber's loss function have been employed. Besides the linear case, SVM's based on polynomials, splines, radial basis function networks and multilayer perceptrons have been successfully applied. Being based on the structural risk minimization principle and capacity concept with pure combinatorial definitions, the quality and complexity of the SVM solution does not depend directly on the dimensionality of the input space (Vapnik, 1995; Vapknik, 1998a; Vapnik, 1998b).

In this paper we formulate a least squares version of SVM's for classification problems with two classes. For the function estimation problem a support vector interpretation of ridge regression (Golub \& Van Loan, 1989) has been given in (Saunders et al., 1998), which considers equality type constraints instead of inequalities from the classical SVM approach. Here, we also consider equality 
constraints for the classification problem with a formulation in least squares sense. As a result the solution follows directly from solving a set of linear equations, instead of quadratic programming. While in classical SVM's many support values are zero (nonzero values correspond to support vectors), in least squares SVM's the support values are proportional to the errors.

This paper is organized as follows. In Section 2 we review some basic work about support vector machine classifiers. In Section 3 we discuss the least squares support vector machine classifiers. In Section 4 examples are given to illustrate the support values and on a two-spiral benchmark problem.

\section{Support Vector Machines for Classification}

In this Section we shortly review some basic work on support vector machines (SVM) for classification problems. For all further details we refer to (Vapnik, 1995; Vapnik, 1998a; Vapnik, 1998b).

Given a training set of $N$ data points $\left\{y_{k}, x_{k}\right\}_{k=1}^{N}$, where $x_{k} \in \mathbb{R}^{n}$ is the $k$ th input pattern and $y_{k} \in \mathbb{R}$ is the $k$ th output pattern, the support vector method approach aims at constructing a classifier of the form:

$$
y(x)=\operatorname{sign}\left[\sum_{k=1}^{N} \alpha_{k} y_{k} \psi\left(x, x_{k}\right)+b\right],
$$

where $\alpha_{k}$ are positive real constants and $b$ is a real constant. For $\psi(\cdot, \cdot)$ one typically has the following choices: $\psi\left(x, x_{k}\right)=x_{k}^{T} x$ (linear SVM); $\psi\left(x, x_{k}\right)=\left(x_{k}^{T} x+1\right)^{d}$ (polynomial SVM of degree $d$ ); $\psi\left(x, x_{k}\right)=\exp \left\{-\left\|x-x_{k}\right\|_{2}^{2} / \sigma^{2}\right\}$ (RBF SVM); $\psi\left(x, x_{k}\right)=\tanh \left[\kappa x_{k}^{T} x+\theta\right]$ (two layer neural SVM), where $\sigma, \kappa$ and $\theta$ are constants.

The classifier is constructed as follows. One assumes that

$$
\begin{array}{ll}
w^{T} \varphi\left(x_{k}\right)+b \geq 1, & \text { if } y_{k}=+1, \\
w^{T} \varphi\left(x_{k}\right)+b \leq-1, & \text { if } y_{k}=-1,
\end{array}
$$

which is equivalent to

$$
y_{k}\left[w^{T} \varphi\left(x_{k}\right)+b\right] \geq 1, \quad k=1, \ldots, N,
$$

where $\varphi(\cdot)$ is a nonlinear function which maps the input space into a higher dimensional space. However, this function is not explicitly constructed. In order to have the possibility to violate (3), in case a separating hyperplane in this higher dimensional space does not exist, variables $\xi_{k}$ are introduced such that

$$
\begin{aligned}
& y_{k}\left[w^{T} \varphi\left(x_{k}\right)+b\right] \geq 1-\xi_{k}, \quad k=1, \ldots, N, \\
& \xi_{k} \geq 0, \quad k=1, \ldots, N .
\end{aligned}
$$

According to the structural risk minimization principle, the risk bound is minimized by formulating the optimization problem

$$
\min _{w, \xi_{k}} g_{1}\left(w, \xi_{k}\right)=\frac{1}{2} w^{T} w+c \sum_{k=1}^{N} \xi_{k}
$$


subject to (4). Therefore, one constructs the Lagrangian

$$
\begin{aligned}
\mathscr{L}_{1}\left(w, b, \xi_{k} ; \alpha_{k}, v_{k}\right)= & g_{1}\left(w, \xi_{k}\right)-\sum_{k=1}^{N} \alpha_{k}\left\{y_{k}\left[w^{T} \varphi\left(x_{k}\right)+b\right]-\right. \\
& \left.-1+\xi_{k}\right\}-\sum_{k=1}^{N} v_{k} \xi_{k}
\end{aligned}
$$

by introducing Lagrange multipliers $\alpha_{k} \geq 0, v_{k} \geq 0(k=1, \ldots, N)$. The solution is given by the saddle point of the Lagrangian by computing

$$
\max _{\alpha_{k}, v_{k}} \min _{w, b, \xi_{k}} \mathcal{L}_{1}\left(w, b, \xi_{k} ; \alpha_{k}, v_{k}\right) .
$$

One obtains

$$
\begin{aligned}
& \frac{\partial \mathcal{L}_{1}}{\partial w_{1}}=0 \rightarrow w=\sum_{k=1}^{N} \alpha_{k} y_{k} \varphi\left(x_{k}\right), \\
& \frac{\partial \mathcal{L}_{1}}{\partial b}=0 \rightarrow \sum_{k=1}^{N} \alpha_{k} y_{k}=0, \\
& \frac{\partial \mathcal{L}_{1}}{\partial \xi_{k}}=0 \rightarrow 0 \leq \alpha_{k} \leq c, k=1, \ldots, N,
\end{aligned}
$$

which leads to the solution of the following quadratic programming problem

$$
\max _{\alpha_{k}} \mathcal{Q}_{1}\left(\alpha_{k} ; \varphi\left(x_{k}\right)\right)=-\frac{1}{2} \sum_{k, l=1}^{N} y_{k} y_{l} \varphi\left(x_{k}\right)^{T} \varphi\left(x_{l}\right) \alpha_{k} \alpha_{l}+\sum_{k=1}^{N} \alpha_{k},
$$

such that

$$
\sum_{k=1}^{N} \alpha_{k} y_{k}=0, \quad 0 \leq \alpha_{k} \leq c, k=1, \ldots, N .
$$

The function $\varphi\left(x_{k}\right)$ in (9) is related then to $\psi\left(x, x_{k}\right)$ by imposing

$$
\varphi(x)^{T} \varphi\left(x_{k}\right)=\psi\left(x, x_{k}\right),
$$

which is motivated by Mercer's Theorem. Note that for the two layer neural SVM, Mercer's condition only holds for certain parameter values of $\kappa$ and $\theta$.

The classifier (1) is designed by solving

$$
\max _{\alpha_{k}} \mathcal{Q}_{1}\left(\alpha_{k} ; \psi\left(x_{k}, x_{l}\right)\right)=-\frac{1}{2} \sum_{k, l=1}^{N} y_{k} y_{l} \psi\left(x_{k}, x_{l}\right) \alpha_{k} \alpha_{l}+\sum_{k=1}^{N} \alpha_{k},
$$

subject to the constraints in (9). One does not have to calculate $w$ nor $\varphi\left(x_{k}\right)$ in order to determine the decision surface. Because the matrix associated with this quadratic programming problem is not indefinite, the solution to (11) will be global (Fletcher, 1987).

Furthermore, one can show that hyperplanes (3) satisfying the constraint $\|w\|_{2} \leq$ $a$ have a VC-dimension $h$ which is bounded by

$$
h \leq \min \left(\left[r^{2} a^{2}\right], n\right)+1,
$$


where [.] denotes the integer part and $r$ is the radius of the smallest ball containing the points $\varphi\left(x_{1}\right), \ldots, \varphi\left(x_{N}\right)$. Finding this ball is done by defining the Lagrangian

$$
\mathcal{L}_{2}\left(r, q, \lambda_{k}\right)=r^{2}-\sum_{k=1}^{N} \lambda_{k}\left(r^{2}-\left\|\varphi\left(x_{k}\right)-q\right\|_{2}^{2}\right),
$$

where $q$ is the center of the ball and $\lambda_{k}$ are positive Lagrange multipliers. In a similar way as for (5) one finds that the center is equal to $q=\sum_{k} \lambda_{k} \varphi\left(x_{k}\right)$, where the Lagrange multipliers follow from

$$
\max _{\lambda_{k}} \mathcal{Q}_{2}\left(\lambda_{k} ; \varphi\left(x_{k}\right)\right)=-\sum_{k, l=1}^{N} \varphi\left(x_{k}\right)^{T} \varphi\left(x_{l}\right) \lambda_{k} \lambda_{l}+\sum_{k=1}^{N} \lambda_{k} \varphi\left(x_{k}\right)^{T} \varphi\left(x_{k}\right),
$$

such that

$$
\sum_{k=1}^{N} \lambda_{k}=1, \quad \lambda_{k} \geq 0, k=1, \ldots, N .
$$

Based on (10), $\mathcal{Q}_{2}$ can also be expressed in terms of $\psi\left(x_{k}, x_{l}\right)$. Finally, one selects a support vector machine with minimal VC dimension by solving (11) and computing (12) from (14).

\section{Least Squares Support Vector Machines}

Here we introduce a least squares version to the SVM classifier by formulating the classification problem as

$$
\min _{w, b, e} \mathcal{g}_{3}(w, b, e)=\frac{1}{2} w^{T} w+\gamma \frac{1}{2} \sum_{k=1}^{N} e_{k}^{2},
$$

subject to the equality constraints

$$
y_{k}\left[w^{T} \varphi\left(x_{k}\right)+b\right]=1-e_{k}, \quad k=1, \ldots, N .
$$

One defines the Lagrangian

$$
\mathcal{L}_{3}(w, b, e ; \alpha)=\mathscr{I}_{3}(w, b, e)-\sum_{k=1}^{N} \alpha_{k}\left\{y_{k}\left[w^{T} \varphi\left(x_{k}\right)+b\right]-1+e_{k}\right\},
$$

where $\alpha_{k}$ are Lagrange multipliers (which can be either positive or negative now due to the equality constraints as follows from the Kuhn-Tucker conditions (Fletcher, 1987)).

The conditions for optimality

$$
\begin{aligned}
& \frac{\partial \mathscr{L}_{3}}{\partial w}=0 \rightarrow w=\sum_{k=1}^{N} \alpha_{k} y_{k} \varphi\left(x_{k}\right), \\
& \frac{\partial \mathscr{L}_{3}}{\partial b_{2}}=0 \rightarrow \sum_{k=1}^{N} \alpha_{k} y_{k}=0, \\
& \frac{\partial \mathcal{L}_{3}}{\partial e_{k}}=0 \rightarrow \alpha_{k}=\gamma e_{k}, k=1, \ldots, N, \\
& \frac{\partial \mathcal{L}_{3}}{\partial \alpha_{k}}=0 \rightarrow y_{k}\left[w^{T} \varphi\left(x_{k}\right)+b\right]-1+e_{k}=0, k=1, \ldots, N
\end{aligned}
$$


can be written immediately as the solution to the following set of linear equations (Fletcher, 1987)

$$
\left[\begin{array}{ccc|c}
I & 0 & 0 & -Z^{T} \\
0 & 0 & 0 & -Y^{T} \\
0 & 0 & \gamma I & -I \\
\hline Z & Y & I & 0
\end{array}\right]\left[\begin{array}{c}
w \\
b \\
e \\
\hline \alpha
\end{array}\right]=\left[\begin{array}{c}
0 \\
0 \\
0 \\
\hline 1
\end{array}\right],
$$

where $Z=\left[\varphi\left(x_{1}\right)^{T} y_{1} ; \ldots ; \varphi\left(x_{N}\right)^{T} y_{N}\right], Y=\left[y_{1} ; \ldots ; y_{N}\right], \overrightarrow{1}=[1 ; \ldots ; 1], e=$ $\left[e_{1} ; \ldots ; e_{N}\right], \alpha=\left[\alpha_{1} ; \ldots ; \alpha_{N}\right]$. The solution is also given by

$$
\left[\begin{array}{c|c}
0 & -Y^{T} \\
\hline Y & Z Z^{T}+\gamma^{-1} I
\end{array}\right]\left[\frac{b}{\alpha}\right]=\left[\frac{0}{1}\right] .
$$

Mercer's condition can be applied again to the matrix $\Omega=Z Z^{T}$, where

$$
\begin{aligned}
\Omega_{k l} & =y_{k} y_{l} \varphi\left(x_{k}\right)^{T} \varphi\left(x_{l}\right) \\
& =y_{k} y_{l} \psi\left(x_{k}, x_{l}\right) .
\end{aligned}
$$

Hence, the classifier (1) is found by solving the linear set of Equations (20)-(21) instead of quadratic programming. The parameters of the kernels such as $\sigma$ for the RBF kernel can be optimally chosen according to (12). The support values $\alpha_{k}$ are proportional to the errors at the data points (18), while in the case of (14) most values are equal to zero. Hence, one could rather speak of a support value spectrum in the least squares case.

\section{Examples}

In a first example (Figure 1) we illustrate the support values for a linearly separable problem of two classes in a two dimensional space. The size of the circles indicated at the training data is chosen proportionally to the absolute values of the support values. A linear SVM has been taken with $\gamma=1$. Clearly, points located close and far from the decision line have the largest support values. This is different from SVM's based on inequality constraints, where only points that are near the decision line have nonzero support values. This can be understood from the fact that the signed distance from a point $x_{k}$ to the decision line is equal to $\left(w^{T} x_{k}+b\right) /\|w\|=$ $\left(1-e_{k}\right) /\left(y_{k}\|w\|\right)$ and $\alpha_{k}=\gamma e_{k}$ in the least squares SVM case.

In a second example (Figure 2) we illustrate a least squares support vector machine RBF classifier on a two-spiral benchmark problem. The training data are shown on Figure 2 with two classes indicated by 'o' and ' $*$ ' (360 points with 180 for each class) in a two dimensional input space. Points in between the training data located on the two spirals are often considered as test data for this problem but are not shown on the figure. The excellent generalization performance is clear from the decision boundaries shown on the figures. In this case $\sigma=1$ and $\gamma=1$ were chosen as parameters. Other methods which have been applied to the two-spiral 


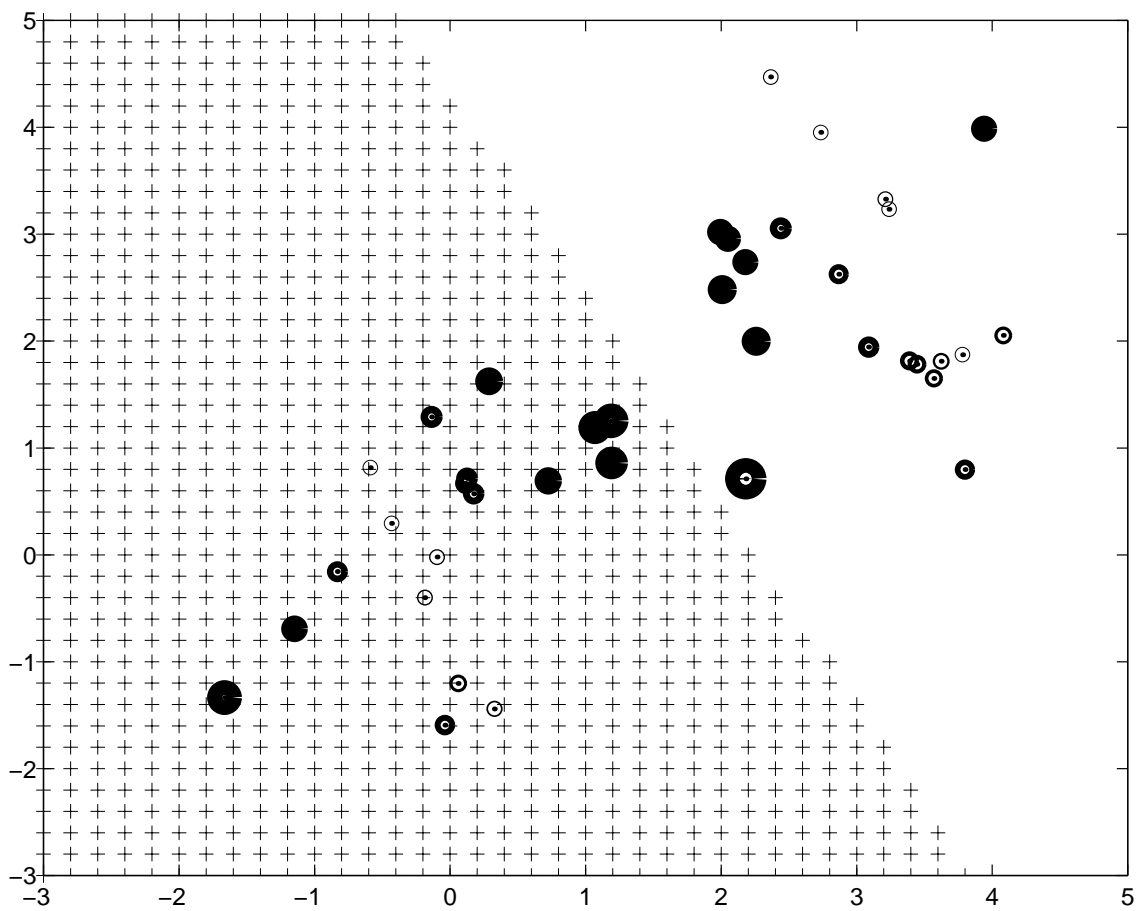

Figure 1. Example of two linearly separable classes in a two-dimensional input space. The size of the circles indicated at the training data is chosen proportionally to the absolute value of the support value.

benchmark problem, such as the use of circular units (Ridella et al., 1997), have shown good performance as well. The least squares SVM solution on the other hand can be found with low computational cost and is free of many local minima, being the solution to a convex optimization problem. For two-spiral classification problems the method gives good results over a wide parameter range of $\sigma$ and $\gamma$ values.

\section{Conclusions}

We discussed a least squares version of support vector machine classifiers. Due to the equality constraints in the formulation, a set of linear equations has to be solved instead of a quadratic programming problem. Mercer's condition is applied as in other SVM's. For a complicated two-spiral classification problem it is illustrated that a least squares SVM with RBF kernel is readily found with excellent generalization performance and low computational cost. 


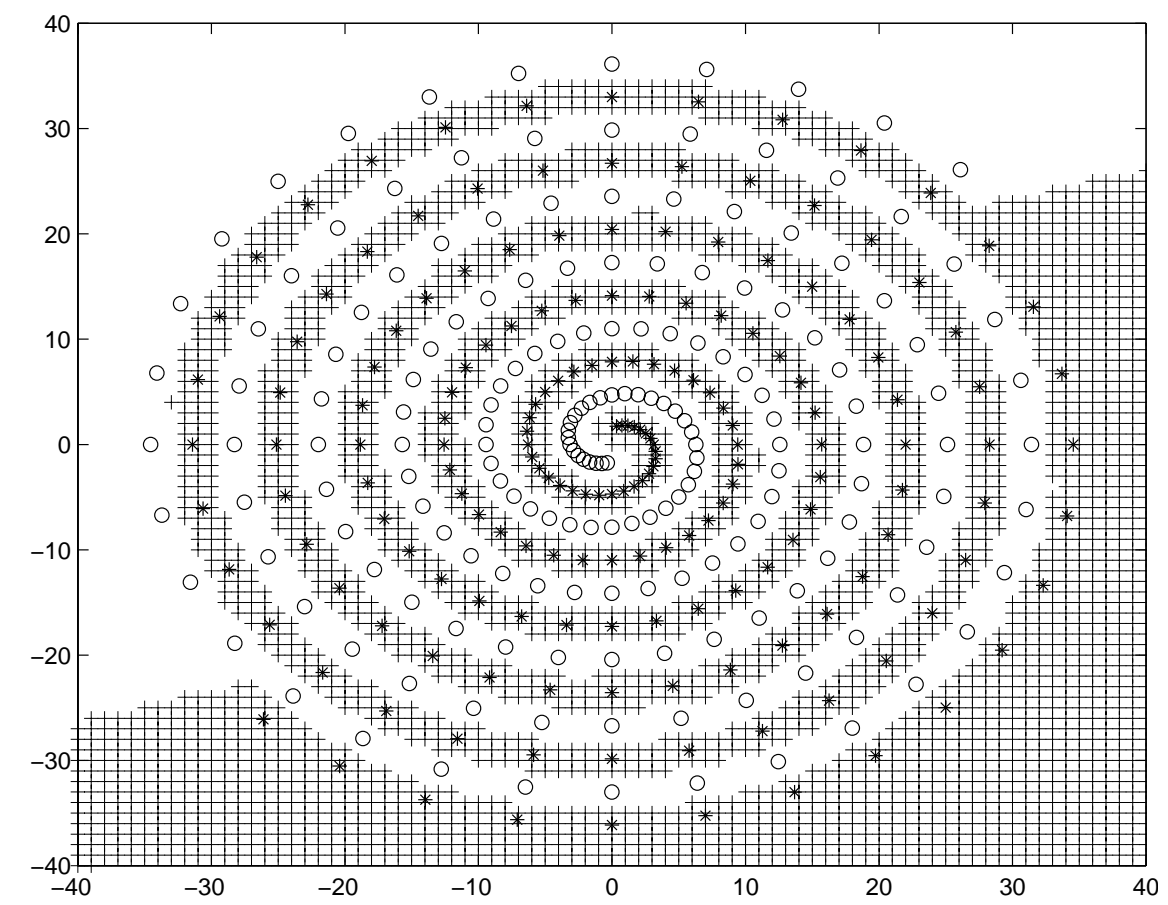

Figure 2. A two-spiral classification problem with the two classes indicated by 'o' and '*' and 180 training data for each class. The figure shows the excellent generalization performance for a least squares SVM machine with RBF kernel.

\section{Acknowledgements}

This research work was carried out at the ESAT laboratory and the Interdisciplinary Center of Neural Networks ICNN of the Katholieke Universiteit Leuven, in the framework of the FWO project G.0262.97 Learning and Optimization: an Interdisciplinary Approach, the Belgian Programme on Interuniversity Poles of Attraction, initiated by the Belgian State, Prime Minister's Office for Science, Technology and Culture (IUAP P4-02 \& IUAP P4-24) and the Concerted Action Project MIPS (Modelbased Information Processing Systems) of the Flemish Community. Johan Suykens is a postdoctoral researcher with the National Fund for Scientific Research FWO - Flanders. We thank Vladimir Vapnik for bringing the work of Saunders et al. to our attention.

\section{References}

1. C.M. Bishop, Neural Networks for Pattern Recognition, Oxford University Press, 1995.

2. V. Cherkassky and F. Mulier, Learning from Data: Concepts, Theory and Methods, John Wiley and Sons, 1998.

3. R. Fletcher, Practical Methods of Optimization, John Wiley and Sons: Chichester and New York, 1987. 
4. G.H. Golub and C.F. van Loan, Matrix Computations, Johns Hopkins University Press: Baltimore MD, 1989.

5. S. Haykin, Neural Networks: A Comprehensive Foundation, Macmillan College Publishing Company: Englewood Cliffs, 1994.

6. S. Ridella, S. Rovetta and R. Zunino, "Circular backpropagation networks for classification," IEEE Transactions on Neural Networks, Vol. 8, No. 1, pp. 84-97, 1997.

7. C. Saunders, A. Gammerman and V. Vovk, "Ridge regression learning algorithm in dual variables," Proceedings of the 15th International Conference on Machine Learning ICML-98, Madison-Wisconsin, 1998.

8. B. Schölkopf, K.-K. Sung, C. Burges, F. Girosi, P. Niyogi, T. Poggio and V. Vapnik, "Comparing support vector machines with Gaussian kernels to radial basis function classifiers," IEEE Transactions on Signal Processing, Vol. 45, No. 11, pp. 2758-2765, 1997.

9. V. Vapnik, "The nature of statistical learning theory," Springer-Verlag: New York, 1995.

10. V. Vapnik, "Statistical learning theory," John Wiley: New York, 1998.

11. V. Vapnik, "The support vector method of function estimation," in J.A.K. Suykens and J. Vandewalle (Eds) Nonlinear Modeling: Advanced Black-Box Techniques, Kluwer Academic Publishers, Boston, pp. 55-85, 1998.

12. J.M. Zurada, Introduction to Artificial Neural Systems, West Publishing Company, 1992. 